\title{
Fractal Model of Normal Contact Stiffness between Two Spheres of Joint Interfaces with Simulation
}

\author{
Jingfang SHEN*, Sha XU**, Wenwei LIU**, Jiajun YANG* \\ *School of Mechanical Science and Engineering, Huazhong University of Science and Technology, 430074 Wuhan, China, \\ E-mail: yjjllar@163.com \\ **College of Science, Huazhong Agricultural University, 430070 Wuhan, China, E-mail: shenjingfang16@126.com \\ cross $^{\text {ref }}$ http://dx.doi.org/10.5755/j01.mech.23.5.19356
}

\section{Introduction}

Normal contact stiffness of joint interfaces is vital to processes ranging from adhesion to friction, wear and lubrication $[1,2]$. It is the fact that the rough surface morphology affects the contact stiffness profoundly, and contact between surfaces is the interaction of asperities. Therefore, the interaction of asperities has been extensively studied.

According to the Hertz solution for a single elastic sphere, Greenwood and Williamson [1] proposed that interaction between contacting asperities was equivalent to contact between a rough surface comprising spherical asperities of constant radius and an ultra-smooth flat surface. Assuming asperity heights follow Gaussian distribution, they gave a statistical model called GW model, a popular method to simulate contact stiffness of joint surface.

Due to the limitation of statistical approach, the GW model relies heavily on statistical roughness parameters of sampling length and resolution of measuring instrument. It means that unbiased information from surface topography is difficult to obtain [2]. Consequently, Majumdar and Tien [2] gave a fractal characterization and simulated rough surfaces to remedy these deficiencies which depended on the scale in the GW model. In addition, Majumdar and Bhushan [3] characterized the multi-scaled self-affine topography by scale-independent parameters, then they proposed a novel mathematical model by using fractal geometry. In their theory, the model (MB model) mainly considered the contact between rough surface and ultra-smooth plane. Additionally, Wang and Komvopoulos [4] analysed the irrationality of MB model and introduced a domain extension of factor associated with fractal dimension to better match real situation. As a result, they established WK contact fractal model with more reasonable and impersonal asperity size distribution function.

Recently, some researchers managed to compute normal contact stiffness by using microscope property of asperities. For example, Zhang [5] proposed a fractal model for normal contact stiffness of machine joint surfaces based on Hertz contact theory between a sphere and a plane under some given hypotheses. It overcame the shortcoming of statistical analysis in the MB model to avoid excessive dependence upon scale. Subsequently, Wen [6] discussed a modified model for normal contact stiffness by using micro-contact size distribution function, as well as a mathematical relationship between normal contact stiffness and characteristic parameters of joint surfaces. Generally, they concluded that normal contact stiffness increased with the normal load and decreased with the fractal characteristic length scale parameter G. Nevertheless, the variation becomes complicated when the fractal dimension D is involved.
Meanwhile, numerous researchers studied the stringency of parameters. For example, Ganti and Bushan [7] doubted the scale-independence of fractal parameters, especially D and G, when they concentrated on the fractal model. It leaded more discussion about fractal parameters [8]. He and Zhu [9] investigated the fractal parameters D and G of two typical kinds of engineering surfaces. The scale-independence of fractal parameters was studied by changing the sampling spacing t corresponding to spatial measuring resolution and the sampling length L. Jiang et al. [10] employed structure function method to calculate the rough surface fractal dimension and scale coefficient, and they indicated that the theoretical contact stiffness of diverse samples is consistent with the experiment data under the different contact load.

Recently, researchers turned their main interests into contact stiffness from different points of views. For example, Ghafoor et al. [11] established a contact stiffness model by using the finite element analysis and gave a novel probability concept in the case of mechanical soft contact. For the most popular GW model, Wu [12] discussed the statistical method of micro-bulges on the one dimensional case. But the prerequisite of their research is that all the microbulges are half spheres and curvature radiuses are equal, which is rather idealized for real situations. Additionally, Robert and Jeffrey [13] adopted multi scale methods to study the contact load between rough surfaces. Persson [14] studied the mechanical properties of the random rough surface. Daniel and Dickrell [15] obtained the contact rigidity from the transverse contact stiffness model, which is consistent with the experiment data under the condition of small load and small contact stress. Filippi and Kartal [16-17] measured the contact stiffness of the joint surfaces.

The research mentioned above involving the fractal model of normal contact stiffness is applicable for a multitude of mechanical joint interface. The relationships between the parameters of fractal dimensions and roughness amplitude are indicated in the mathematical formulas and simulation. Furthermore, another benefit is the convenience of calculating.

However, from the literature, these models have a shared problem that they are all based on MB fractal model. This means that the contact of two rough surfaces is simplified to a rigid plane and a single micro-bulge which is equivalent to half a sphere [18-21]. Obviously, the model has its limitation. For example, some of these works are confined to pure elastic deformation of the contacting sphere [22-31]. An attempt to solve this problem is given by L. Kogut and I. Etsion [32]. They presented general solutions for the elastic-plastic contact, but it still aimed at a deformable sphere and a rigid flat. 
It is well-known that joint interfaces are constituted by two rough surfaces. For the single micro-bulge of the rough surfaces, they could be regarded as equivalent spheres with curvature radius $R$. Thus, the two micro-bulge of the rough surfaces are equivalent to two different half spheres. In this paper, the main objective of our research is to offer a new fractal model of normal contact stiffness which is more universal than before. Meanwhile, the evolution of the elastic-plastic contact is analysed involving three distinct stages which range from complete elastic through elastic-plastic to fully plastic deformation. Moreover, we could find that the previous model is only one of the special cases.

\section{Normal contact stiffness model of the joint surface}

\subsection{The contact of two spheres}

\subsubsection{Perfectly elastic deformation stage}

Joint surface essentially composed of two rough surfaces could be simplified to a rough surface and a true plane [33-35]. This paper considering system of contact model of two rough surface fractal characteristics is in order to make contact more general. Two convex bodies of the rough surface contact are equivalent to two hemispheres.

Let $R_{1}$ and $R_{2}$ be the radius of the two half spheres. The two hemispheres just contact on the point $\mathrm{O}$ when no load is applied. The state is as shown in Fig. 1.

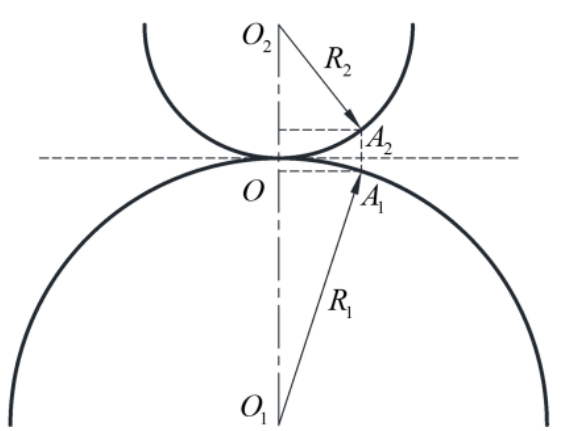

Fig. 1 Two convex bodies of the rough surface

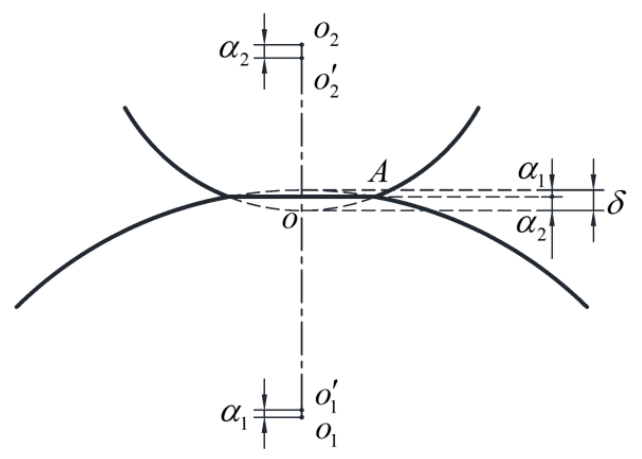

Fig. 2 Two spheres normal contact deformation

When the two hemispheres are under the action of the normal load $\mathrm{p}$, two spheres will produce normal contact deformation, the normal contact deformation of the first hemisphere is $\alpha_{1}$ and the second one is $\alpha_{2}$, then the total normal contact deformation is $\delta=\alpha_{1}+\alpha_{2}$. The contact state of the two hemispheres is as shown in Fig. 2.

In engineering practice, it is a common phenomenon that two curved objects contact each other to transfer pressure. For example, the contact between the ball of rolling bearing and its seat, wheel and rail, etc. Before loading, these contacts are seemed to be point contacts or line contacts. Due to the material deformation after loading, these point or line contacts become contact surfaces. There are large pressures on the small convex surface and area nearby. Considering contact problem, it is often assumed that surface is rigid smooth. Based on the analysis of deformation, subjecting to the contact distribution of the deformation, the maximum compressive stress on contact surface is proposed.

According to Hertz theory, the relationship between the normal contact deformation $\delta$ and the normal load $\mathrm{p}$ [36] can be expressed as:

$$
\delta=\left(\frac{9 \pi^{2} p^{2}\left(k_{1}+k_{2}\right)^{2}\left(R_{1}+R_{2}\right)}{16 R_{1} R_{2}}\right)^{\frac{1}{3}},
$$

where $R_{1}$ and $R_{2}$ are the equivalent curvature radius of the two hemispheres, $k_{1}$ and $k_{2}$ satisfy the equation $k_{1}=\left(1-v_{1}^{2}\right) /\left(\pi E_{1}\right), k_{2}=\left(1-v_{2}^{2}\right) /\left(\pi E_{2}\right)$, which $E_{1}$ and $E_{2}$ are the elastic modulus of two contact materials, $v_{1}$ and $v_{2}$ are the corresponding Poisson ratio.

Then the relation between normal load $\mathrm{p}$ and normal contact deformation $\delta$ can be given as:

$$
\begin{aligned}
& p=\frac{4 \delta^{\frac{3}{2}}}{3 \pi\left(k_{1}+k_{2}\right)}\left(\frac{R_{1} R_{2}}{R_{1}+R_{2}}\right)^{\frac{1}{2}}, \\
& k_{1}+k_{2}=\frac{1}{\pi E},
\end{aligned}
$$

where $E$ is the equivalent elastic modulus of the two materials and it satisfies:

$$
E=\left(1-v_{1}^{2}\right) / E_{1}+\left(1-v_{2}^{2}\right) / E_{2} \text {. }
$$
we obtain:

Substituting from equation (3) into equation (2),

$$
p_{e}=\frac{4}{3} E \sqrt{\frac{R_{1} R_{2}}{R_{1}+R_{2}}} \delta^{\frac{3}{2}},
$$

where $p_{e}$ is complete elastic contact load.

The normal contact stiffness $k_{e}$ of two asperities is given as:

$$
k_{e}=\frac{\partial p_{e}}{\partial \delta}
$$

Substituting $p_{e}$ from equation (4) into the equation above,

$$
k_{e}=2 E \sqrt{\frac{R_{1} R_{2}}{R_{1}+R_{2}}} \delta^{\frac{1}{2}} .
$$


We will discuss two cases as following, one is $R_{1}<R_{2}$, and the other is $R_{1}=R_{2}$. Obviously, the case of $R_{1}>R_{2}$ is similar to the one of $R_{1}<R_{2}$. The occasion of $R_{1}<<R_{2}$ is similar to the contact between an asperity and a flat plane [37].

I. When $R_{1}<R_{2}$, because the deformation of the smaller hemisphere is stronger than the other during contacting, we choose the sectional area of the smaller one. Based on the geometrical relationship of asperities before deformation, we consider $R_{1} \gg \delta$, we can get:

$$
a^{\prime}=2 \pi R_{1} \delta
$$

where $a^{\prime}$ is sectional area of micro-contact with curvature radius $R_{1}$. So the normal contact deformation can be given as:

$$
\delta=\frac{a^{\prime}}{2 \pi R_{1}} .
$$
obtain:

Using $\delta$ from equation (8) into equation (6), we

$$
k_{e}=2 E \sqrt{\frac{R_{2}}{2 \pi\left(R_{1}+R_{2}\right)}} a^{\frac{1}{2}} .
$$

\subsubsection{Perfectly plastic deformation stage}

When $\delta \geq 76.4 \delta_{c}$ the contact of asperities entry a range of complete plastic deformation, where $\delta_{c}$ is the critical deformation when micro contact turns from perfectly elastic stage to elastic-plastic transition deformation. Depending on the fully plastic deformation mechanism, we can express the contact load as [37]:

$$
p_{p}=2 H \pi R \delta \text {. }
$$

The critical deformation $\delta_{c}$ for the inception of plastic contact is given by $[33,34]$ :

$$
\delta_{c}=\left(\frac{\pi k H}{2 E}\right)^{2} R,
$$

where $R$ is the equivalent radius of curvature, $H$ is the hardness of soft material, both of $H$ and $Y$ satisfy $H=2.8 Y$ in which $Y$ is the yield strength of softer materials , $k$ is the hardness coefficient whose value is associated with the Poisson Ratio and $k=0.454+0.41 v$.

\subsubsection{The elastic-plastic transition deformation stage}

When $\delta_{c} \leq \delta \leq 76.4 \delta_{c}$, the contact is in the stage of elastic-plastic transition deformation. The contact load of this stage is given as [39]:

$$
p_{e p}=\frac{2}{3} \times k H \pi R \delta\left(\frac{\delta}{\delta_{c}}\right)^{1.38} .
$$

Now substituting equation (17) and (12) into equation (5), the normal contact stiffness can be expressed as:

$$
\begin{aligned}
& k_{e p}=\frac{d_{e p}}{d \delta}=\frac{2}{3} \times 2.38 k H \pi R\left(\frac{\delta}{\delta_{c}}\right)^{1.38}= \\
& =\frac{2}{3} \times 2.38 k H \pi \cdot \frac{a^{\prime \frac{D}{2}}}{\pi^{2} G^{D-1}}\left(\frac{a_{c}^{\prime}}{a^{\prime}}\right)^{1.38(D-1)}= \\
& =\frac{2}{3} \times \frac{2.38 k H a_{c}^{\prime 1.38(D-1)} a^{\prime 1.38-0.88 D}}{\pi G^{D-1}} .
\end{aligned}
$$

2. 2. The elastic-plastic fractal model of normal contact stiffness of joint surface

The normal deformation of asperity on joint surface will be in a fully plastic deformation stage when $\delta \geq 76.4 \delta_{c}$. That is, there exists normal contact stiffness in the other stages, complete elastic and elastic-plastic deformation.

The curvature radius of the asperity can be given as:

$$
R_{1}=\frac{a^{\frac{D}{2}}}{\pi^{2} G^{D-1}}
$$

where $G$ is the characteristic length scale parameter and $D$ is fractal dimension.

From equation (6), the $\delta$ can be obtained as:

$$
\delta=\frac{k_{e}^{2}}{4 E^{2} \times \frac{R_{1} R_{2}}{R_{1}+R_{2}}} .
$$

Substituting $k_{e}$ from equation (9) and $R_{1}$ from equation (14) into equation (15), yields:

$$
\delta=\frac{\pi}{2} G^{D-1} a^{\frac{2-D}{2}}
$$

Comparing the equation (11), (14), (15), the relation of $\delta$ and $\delta_{c}$ can be rewrote as:

$$
\frac{\delta}{\delta_{c}}=\left(\frac{a_{c}{ }^{\prime}}{a^{\prime}}\right)^{D-1},
$$

where $a_{c}{ }^{\prime}$ is the critical truncated area of single asperity turns from perfectly elastic deformation into elastic-plastic transition deformation stage. And the expression can be shown as:

$$
a_{c}{ }^{\prime}=\left(2 \pi G^{2(D-1)}\left(\frac{E}{k H}\right)^{2}\right)^{\frac{1}{D-1}} .
$$

In order to calculate cross-sectional area of normal load from the critical contact area to the largest area accurately, the size distribution function was given as: 


$$
n\left(a^{\prime}\right)=\frac{D}{2} \psi^{\frac{2-D}{2}} a_{l}^{, \frac{D}{2}} a^{-, \frac{D+2}{2}}
$$

where $\psi$ is the domain extension factor and $\psi>1$.

The normal contact stiffness of the whole joint surface can be shown as:

$$
\begin{aligned}
& K_{n}=\int_{76.4^{1-D}}^{a_{c^{\prime}}} a_{a^{\prime}} k_{e p} n\left(a^{\prime}\right) d a^{\prime}+\int_{a_{c}}^{a_{l^{\prime}}{ }^{\prime}} k_{e} n\left(a^{\prime}\right) d a^{\prime}= \\
& =\frac{2.38 \times 2.8 D k Y\left(1-76.4^{1.38}\right) \psi^{\frac{2-D}{2}} a_{l}{ }^{\frac{D}{2}}}{3 \times 1.38 \pi(1-D) G^{D-1}}+ \\
& \frac{+2 E D \psi^{\frac{2-D}{2}} \sqrt{\frac{R_{2}}{2 \pi\left(R_{1}+R_{2}\right)}} a_{l} l^{\frac{D}{2}}\left(a_{l}{ }^{\frac{1-D}{2}}-a_{c}{ }^{\frac{1-D}{2}}\right)}{1-D} .
\end{aligned}
$$

The relation between cross-sectional area of the elastic contact $a^{\prime}$ and the actual contact area $a$ is given as [18]:

$$
a^{\prime}=2 a, a_{l}{ }^{\prime}=2 a_{l}=\frac{2(2-D)}{D \psi^{\frac{2-D}{2}}} A_{r}, a_{c}{ }^{\prime}=2 a_{c},
$$

where $a_{l}{ }^{\prime}$ is cross-sectional area of the largest micro contact, $a$ is actual contact area of the largest contact point, $A_{r}$ is the total actual contact area.

Using the equation (21) into the equation (20) we obtain:

$$
\begin{aligned}
K_{n}= & \frac{2.38 \times 2.8 D k Y\left(1-76.4^{1.38}\right) \psi^{\frac{2-D}{2}}}{3 \times 1.38 \pi(1-D) G^{(D-1)}}\left(\frac{2(2-D)}{D \psi^{\frac{2-D}{2}}} A_{r}\right)^{\frac{D}{2}}+ \\
& +\frac{2 E D \psi^{\frac{2-D}{2}} \sqrt{\frac{R_{2}}{2 \pi\left(R_{1}+R_{2}\right)}}\left(\frac{2(2-D)}{1-D}\right)^{\frac{D}{2}} \times}{\left.\left.D \psi^{\frac{2-D}{2}}\right)^{\frac{1-D}{2}}-\left(2 a_{c}\right)^{\frac{1-D}{2}}\right\} .} \\
& \times\left\{\left(\frac{2(2-D)}{D \psi^{\frac{2-D}{2}}} A_{r}\right)^{\frac{1-D}{2}}\right.
\end{aligned}
$$

So the dimensionless total normal contact stiffness $K_{n}{ }^{*}$ can be written as:

$$
\begin{aligned}
K_{n}^{*}= & \frac{2.38 \times 2.8 D k \phi\left(1-76.4^{1.38}\right) \psi^{(2-D) / 2}\left(\frac{2(2-D)}{D \psi^{(2-D) / 2}} A_{r}^{*}\right)^{D / 2}}{3 \times 1.38 \pi(1-D) G^{(D-1)}}+\frac{2 D \psi^{(1-D) / 2} \sqrt{\frac{R_{2}}{2 \pi\left(R_{1}+R_{2}\right)}}\left(\frac{2(2-D)}{D \psi^{(2-D) / 2}} A_{r}^{*}\right)^{D / 2} \times}{1-D} \times \\
& \times\left\{\left(\frac{2(2-D)}{D \psi^{(2-D) / 2}} A_{r}^{*}\right)^{(1-D) / 2}-\left(2 a_{c}^{*}\right)^{(1-D) / 2}\right\},
\end{aligned}
$$

where $G^{*}$ is dimensionless characteristic length scale parameter, $A_{r}{ }^{*}$ is dimensionless real contact area, $a_{c}{ }^{*}$ is dimensionless critical contact area. $A_{a}$ is nominal contact area and $\phi$ is plasticity index. They are written as:

$$
K_{n}{ }^{*}=\frac{K_{n}}{E \sqrt{A_{a}}}, G^{*}=\frac{G}{\sqrt{A_{a}}}, A_{r}^{*}=\frac{A_{r}}{A_{a}}, a_{c}{ }^{*}=\frac{a_{c}}{A_{a}}, \phi=\frac{Y}{E} .
$$

\section{The elastic-plastic fractal model of normal contact load of joint surface}

Substituting $p_{e}$ from equation (9) into equation where the completely elastic contact load of the joint surface can be given as:

$$
\begin{aligned}
& P_{e}=\int_{a_{c}}^{a_{l}{ }^{\prime}} p_{e} n\left(a^{\prime}\right) d a^{\prime}= \\
& =\frac{\sqrt{2 \pi} E D G^{(D-1)} \psi^{\frac{2-D}{2}}}{3(3-2 D)} \sqrt{\frac{R_{2}}{R_{1}+R_{2}}} a_{l}{ }^{\frac{D}{2}} \times \\
& \times\left(a_{l}{ }^{\frac{3-2 D}{2}}-a_{c}{ }^{\frac{3-2 D}{2}}\right), D \neq 1.5,
\end{aligned}
$$

$$
P_{e}=2^{-\frac{3}{4}} \sqrt{\pi} E G^{\frac{1}{2}} \psi^{\frac{1}{4}} a_{l}{ }^{\prime^{\frac{3}{4}}} \sqrt{\frac{R_{2}}{R_{1}+R_{2}}} \ln \frac{a_{l}{ }^{\prime}}{a_{c}{ }^{\prime}}, D=1.5 .
$$

Using $p_{e}$ from Eq. (10), the completely plastic contact load on joint surface can be given as following:

$$
\begin{aligned}
& P_{p}=\int_{0}^{76.4^{\frac{1}{1-D}} a_{c}} p_{p} n\left(a^{\prime}\right) d a^{\prime}= \\
& =\frac{5.6 Y D \psi^{\frac{2-D}{2}} a_{l}^{\frac{D}{2}} 76.4^{\frac{2-D}{2(1-D)}} a_{c}^{{ }^{\prime 2}}}{2(2-D)} .
\end{aligned}
$$

Substituting $p_{e p}$ from Eq. (12), we can get the elastic-plastic contact load on joint surface:

$$
\begin{aligned}
& P_{e p}=\int_{76.4^{\frac{1-D}{1-D}}}^{a_{c^{\prime}}{ }^{\prime}} p_{e p} n\left(a^{\prime}\right) d a^{\prime}= \\
& =\frac{D}{2} \psi^{\frac{2-D}{2}} a_{l}{ }^{\frac{D}{2}} \frac{2.8\left(1-76.4^{\frac{2.38-1.88 D}{1-D}}\right) k Y a_{c}{ }^{\frac{2-D}{2}}}{3(2.38-1.88 D)} .
\end{aligned}
$$


When $D \neq 1.5$, the total normal load can be shown as:

$$
\begin{aligned}
& P=P_{e}+P_{p}+P_{e p}=\frac{D}{2} \psi^{\frac{2-D}{2}} a_{l}\left(\frac{5.6 Y \cdot 76.4^{\frac{2-D}{2(1-D)}} a_{c}{ }^{\frac{2-D}{2}}}{2-D}+\right. \\
& \left.+\frac{2.8\left(1-76.4^{\frac{2.38-1.88 D}{1-D}}\right) k Y a_{c}{ }^{\frac{2-D}{2}}}{3(2.38-1.88 D)}+\frac{2 \sqrt{2 \pi} E G^{(D-1)}}{3(3-2 D)} \sqrt{\frac{R_{2}}{R_{1}+R_{2}}}\left(a_{l}{ }^{\frac{3-2 D}{2}}-a_{c}{ }^{\frac{3-2 D}{2}}\right)\right)
\end{aligned}
$$

Then we combine the formula (21) into the formula (27) and get the equation as:

$$
\begin{aligned}
P & =\frac{D}{2} \psi^{\frac{2-D}{2}}\left(\frac{2(2-D)}{D \psi^{\frac{2-D}{2}}} A_{r}\right)^{\frac{D}{2}} \times\left(\frac{5.6 Y \cdot 76.4^{\frac{2-D}{2(1-D)}} \cdot\left(2 a_{c}\right)^{\frac{2-D}{2}}}{2-D}+\frac{2.8\left(1-76.4^{\frac{2.38-1.88 D}{1-D}}\right) k Y\left(2 a_{c}\right)^{\frac{2-D}{2}}}{3(2.38-1.88 D)}+\right. \\
& \left.+\frac{2 \sqrt{2 \pi} E G^{(D-1)}}{3(3-2 D)} \sqrt{\frac{R_{2}}{R_{1}+R_{2}}}\left(\left(\frac{2(2-D)}{D \psi^{\frac{2-D}{2}}} A_{r}\right)^{\frac{3-2 D}{2}}-\left(2 a_{c}\right)^{\frac{3-2 D}{2}}\right)\right)
\end{aligned}
$$

Using the total normal load in a non-dimensional form $P^{*}$ as:

$$
\begin{aligned}
P^{*}= & \frac{D}{2} \psi^{\frac{2-D}{2}}\left(\frac{2(2-D)}{D \psi^{\frac{2-D}{2}}} A_{r}^{*}\right)^{\frac{D}{2}} \times\left(\frac{5.6 \varphi \cdot 76.4^{\frac{2-D}{2(1-D)}} 2^{\frac{2-D}{2}} a_{c}^{* \frac{2-D}{2}}}{2-D}+\frac{2.8\left(1-76.4^{\frac{2.38-1.88 D}{1-D}}\right) k \phi\left(2 a_{c}^{*}\right)^{\frac{2-D}{2}}}{3(2.38-1.88 D)}+\right. \\
& \left.+\frac{2 \sqrt{2 \pi} E G^{*(D-1)}}{3(3-2 D)} \sqrt{\frac{R_{2}}{R_{1}+R_{2}}}\left(\left(\frac{2(2-D)}{D \psi^{\frac{2-D}{2}}} A_{r}^{*}\right)^{\frac{3-2 D}{2}}-\left(2 a_{c}^{*}\right)^{\frac{3-2 D}{2}}\right)\right),
\end{aligned}
$$

where $P^{*}=\frac{P}{E A_{a}}, G^{*}=\frac{G}{\sqrt{A_{a}}}, A_{r}{ }^{*}=\frac{A_{r}}{A_{a}}, a_{c}{ }^{*}=\frac{a_{c}}{A_{a}}$.

If $D=1.5$,

$$
\begin{aligned}
& P_{e}=2^{-\frac{3}{4}} \sqrt{\pi} E G^{\frac{1}{2}} \psi^{\frac{1}{4}} a_{l}{ }^{\frac{3}{4}} \sqrt{\frac{R_{2}}{R_{1}+R_{2}}} \ln \frac{a_{l}{ }^{\prime}}{a_{c}{ }^{\prime}}, \\
& P_{p}=5.6 Y \frac{3}{2} \psi^{\frac{1}{4}} a_{l}{ }^{\frac{3}{4}} 76.4^{-2} a_{c}{ }^{\frac{1}{4}}, \\
& P_{e p}=\frac{3}{4} \psi^{\frac{1}{4}} a_{l}^{\prime \frac{3}{4}} \frac{2.8\left(1-76.4^{-0.12}\right) k Y a_{c}{ }^{\prime \frac{1}{4}}}{0.18} .
\end{aligned}
$$

We can get the total normal load as:

$$
\begin{aligned}
& P=P_{e}+P_{p}+P_{e p}, \\
& P=\psi^{\frac{1}{4}} a_{l}{ }^{\frac{3}{4}}\left(2^{-\frac{3}{4}} \sqrt{\pi} E G^{\frac{1}{2}} \sqrt{\frac{R_{2}}{R_{1}+R_{2}}} \ln \frac{a_{l}{ }^{\prime}}{a_{c}{ }^{\prime}}+\right. \\
& \left.+5.6 Y \frac{3}{2} 76.4^{-2} a_{c}{ }^{\frac{1}{4}}+\frac{3}{4} \cdot \frac{2.8\left(1-76.4^{-0.12}\right) k Y a_{c}{ }^{\prime \frac{1}{4}}}{0.18}\right),
\end{aligned}
$$

$$
\begin{aligned}
& P=\psi^{\frac{1}{4}}\left(\frac{2}{3 \psi^{\frac{1}{4}}} A_{r}\right)^{\frac{3}{4}}\left(2^{-\frac{3}{4}} \sqrt{\pi} E G^{\frac{1}{2}} \sqrt{\frac{R_{2}}{R_{1}+R_{2}}} \ln \frac{A_{r}}{3 \psi^{\frac{1}{4}} a_{c}}+\right. \\
& \left.+5.6 Y \frac{3}{2} 76.4^{-2}\left(2 a_{c}\right)^{\frac{1}{4}}+\frac{3}{4} \frac{2.8\left(1-76.4^{-0.12}\right) k Y\left(2 a_{c}\right)^{\frac{1}{4}}}{0.18}\right) .
\end{aligned}
$$
form as:

Giving the total normal load in a non-dimensional

$$
\begin{aligned}
& P^{*}=\psi^{\frac{1}{4}}\left(\frac{2}{3 \psi^{\frac{1}{4}}} A_{r}^{*}\right)^{\frac{3}{4}}\left(2^{-\frac{3}{4}} \sqrt{\pi} G^{* \frac{1}{2}} \sqrt{\frac{R_{2}}{R_{1}+R_{2}}} \ln \frac{A_{r}^{*}}{3 \psi^{\frac{1}{4}} a_{c}^{*}}+\right. \\
& \left.+5.6 \phi \frac{3}{2} 76.4^{-2}\left(2 a_{c}^{*}\right)^{\frac{1}{4}}+\frac{3}{4} \frac{2.8\left(1-76.4^{-0.12}\right) k \phi\left(2 a_{c}^{*}\right)^{\frac{1}{4}}}{0.18}\right)
\end{aligned}
$$

where $P^{*}=\frac{P}{E A_{a}}, G^{*}=\frac{G}{\sqrt{A_{a}}}, A_{r}^{*}=\frac{A_{r}}{A_{a}}, a_{c}{ }^{*}=\frac{a_{c}}{A_{a}}$.

Because $K_{n}{ }^{*}$ and $P^{*}$ are the function of $A_{r}{ }^{*}$, from the equation (22),(29),(35), we build the implicit function relationship of $K_{n}{ }^{*}$ and $P^{*}$, which is a complicated non-linear relationship.

$$
\text { II. When: }
$$

$$
R_{1}=R_{2}=R \text {. }
$$


Put equation (36) into the equation (1) to get the relation of $\delta$ :

$$
\delta=\left(\frac{9 \pi^{2} p^{2}\left(k_{1}+k_{2}\right)^{2}}{8 R}\right)^{\frac{1}{3}}
$$

Through the deformation of equation (37), the relation between normal contact deformation $\delta$ and normal load $\mathrm{p}$ can be expressed as:

$$
p=\frac{2 \sqrt{2} R^{\frac{1}{2}}}{3 \pi\left(k_{1}+k_{2}\right)} \delta^{\frac{3}{2}}
$$

Substituting for $k_{1}+k_{2}$ from equation (3) in equation (38):

$$
P_{e}=\frac{2 \sqrt{2}}{3} E R^{\frac{1}{2}} \delta^{\frac{3}{2}}
$$

Based on equation (5) and equation (39), we obtain:

$$
k_{e}=\sqrt{2} E R^{\frac{1}{2}} \delta^{\frac{1}{2}}
$$

Based on the geometrical relationship of microbluge before deformation and the typical value of fractal roughness parameters $\mathrm{G}$, we consider $R_{1} \gg \delta$. Then we can get the relationship between $a^{\prime}$ and $R_{1}$, which is:

$$
a^{\prime}=2 \pi R_{1} \delta
$$

Through the deformation of equation (41), the relation between $a^{\prime}$ and $R_{1}$ can be given as:

$$
\delta=\frac{a^{\prime}}{2 \pi R_{1}}
$$

Using equation (42) and equation (40), the relation of normal contact stiffness of two micro convex bodies $k_{e}$ and $a^{\prime}$ can be expressed as:

$$
k_{e}=\sqrt{2} E \sqrt{\frac{a^{\prime}}{2 \pi}} .
$$

The normal contact stiffness on elastic-plastic stage can be given as:

$$
\begin{aligned}
& k_{e p}=\frac{d p_{e p}}{d \delta}=\frac{2}{3} \times 2.38 k H \pi R\left(\frac{\delta}{\delta_{c}}\right)^{1.38}= \\
& =\frac{2}{3} \times \frac{2.38 k H a_{c}{ }^{1.38(D-1)} a^{1.38-0.88 D}}{\pi G^{D-1}} .
\end{aligned}
$$

The normal contact stiffness of the total joint surface can be obtained as:

$$
\begin{aligned}
& K_{n}=\int_{76.4^{\frac{1-D}{1-D}}}^{a_{c}{ }^{\prime}} k_{c^{\prime}} k_{e p} n\left(a^{\prime}\right) d a^{\prime}+\int_{a_{c}^{\prime}}^{a_{l^{\prime}}} k_{e} n\left(a^{\prime}\right) d a^{\prime}= \\
& =\frac{2.38 \times 2.8 D k Y\left(1-76.4^{1.38}\right) \psi^{\frac{2-D}{2}} a_{l}{ }^{\frac{D}{2}}}{3 \times 1.38(1-D) \pi G^{D-1}}+ \\
& +\frac{D E \psi^{\frac{2-D}{2}} a_{l}{ }^{\frac{D}{2}}}{(1-D) \sqrt{\pi}}\left(a_{l}{ }^{\frac{1-D}{2}}-a_{c}{ }^{\frac{1-D}{2}}\right) .
\end{aligned}
$$

Finally, we can have the final form of the total normal contact stiffness:

$$
\begin{aligned}
& K_{n}=\frac{2.38 \times 2.8 D k Y\left(1-76.4^{1.38}\right) \psi^{\frac{2-D}{2}}\left(\frac{2(2-D)}{D \psi^{\frac{2-D}{2}}} A_{r}\right)^{\frac{D}{2}}}{3 \times 1.38(1-D) \pi G^{(D-1)}}+ \\
& +\frac{D E \psi^{\frac{2-D}{2}}\left(\frac{2(2-D)}{D \psi^{\frac{2-D}{2}}} A_{r}\right)^{\frac{D}{2}}}{(1-D) \sqrt{\pi}} \cdot\left(\left(\frac{2(2-D)}{D \psi^{\frac{2-D}{2}}} A_{r}\right)^{\frac{1-D}{2}}-\left(2 a_{c}\right)^{\frac{1-D}{2}}\right),
\end{aligned}
$$

where $a^{\prime}=2 a, a_{l}{ }^{\prime}=2 a_{l}=\frac{2(2-D)}{D \psi^{\frac{2-D}{2}}} A_{r}, a_{c}{ }^{\prime}=2 a_{c}$.

Give the total normal contact stiffness in a non-dimensional form as:

$$
\begin{aligned}
& K_{n}{ }^{*}=\frac{2.38 \times 2.8 D k \phi\left(1-76.4^{1.38}\right) \psi^{\frac{2-D}{2}}\left(\frac{2(2-D)}{D \psi^{\frac{2-D}{2}}} A_{r}^{*}\right)^{\frac{D}{2}}}{3 \times 1.38(1-D) \pi G^{*(D-1)}}+ \\
& +\frac{D \psi^{\frac{2-D}{2}}\left(\frac{2(2-D)}{D \psi^{\frac{2-D}{2}}} A_{r}^{*}\right)^{\frac{D}{2}}}{(1-D) \sqrt{\pi}} \cdot\left(\left(\frac{2(2-D)}{D \psi^{\frac{2-D}{2}}} A_{r}^{*}\right)^{\frac{1-D}{2}}-\left(2 a_{c}^{*}\right)^{\frac{1-D}{2}}\right),
\end{aligned}
$$

where

$$
K_{n}^{*}=\frac{K_{n}}{E \sqrt{A_{a}}}, G^{*}=\frac{G}{\sqrt{A_{a}}}, A_{r}^{*}=\frac{A_{r}}{A_{a}}, a_{c}{ }^{*}=\frac{a_{c}}{A_{a}}, \phi=\frac{Y}{E} \text {. }
$$

If $D \neq 1.5$, substituting for $p_{e}$ from equation(39) in equation where the completely elastic contact load of the joint surface can be given as following:

$$
\begin{aligned}
P_{e} & =\int_{a_{c}^{\prime}}^{a_{l}^{\prime}} p_{e} n\left(a^{\prime}\right) d a^{\prime}= \\
& =\frac{\sqrt{\pi} E G^{(D-1)} D \psi^{\frac{2-D}{2}}}{3(3-2 D)} a_{l}^{\prime \frac{D}{2}}\left(a_{l}{ }^{\frac{3-2 D}{2}}-a_{c} \frac{3-2 D}{2}\right) .
\end{aligned}
$$

Substituting for $p_{p}$ from equation (10) in equation where the completely plastic contact load of the joint surface can be obtained as: 


$$
\begin{aligned}
P_{p} & =\int_{0}^{76.4^{\frac{1}{1-D}} a_{c}{ }^{\prime}} p_{p} n\left(a^{\prime}\right) d a^{\prime}= \\
& =\frac{5.6 Y D \psi^{\frac{2-D}{2}} a_{l}{ }^{\frac{D}{2}} 76.4^{\frac{2-D}{2(1-D)}} a_{c}{ }^{\frac{2-D}{2}}}{2(2-D)} .
\end{aligned}
$$

Substituting for $p_{e p}$ from equation (12) in equa-

tion where the elastic-plastic contact load of the joint surface can be expressed as:

$$
\begin{aligned}
P_{e p} & =\int_{76.4^{1-D}}^{a_{c^{\prime}}} a_{c^{\prime}} p_{e p} n\left(a^{\prime}\right) d a^{\prime}= \\
& =\frac{D}{2} \psi^{\frac{2-D}{2}} a_{l}{ }^{\frac{D}{2}} \frac{2.8\left(1-76.4^{\frac{2.38-1.88 D}{1-D}}\right) k Y a_{c}{ }^{\frac{2-D}{2}}}{3(2.38-1.88 D)} .
\end{aligned}
$$

Therefore, we have the total normal contact load of the joint surface:

$P=P_{e}+P_{p}+P_{e p}=\frac{D}{2} \psi^{\frac{2-D}{2}} a_{l}{ }^{\frac{D}{2}}\left(\frac{2 \sqrt{\pi} E G^{D-1}}{3(3-2 D)}\left(a_{l}{ }^{\frac{3-2 D}{2}}-a_{c}{ }^{\frac{3-2 D}{2}}\right)+\frac{5.6 Y \cdot 76.4^{\frac{2-D}{2(1-D)}} a_{c}{ }^{\frac{2-D}{2}}}{2-D}+\right.$

$$
\left.+\frac{2.8\left(1-76.4^{\frac{2.38-1.88 D}{1-D}}\right) k Y a_{c}{ }^{\frac{2-D}{2}}}{3(2.38-1.88 D)}\right) .
$$

Combine the equation (21) and the equation (51) to get the final form:

$$
\begin{aligned}
& P=\frac{D}{2} \psi^{\frac{2-D}{2}}\left(\frac{2(2-D)}{D \psi^{\frac{2-D}{2}}} A_{r}\right)^{\frac{D}{2}}\left(\frac{2 \sqrt{\pi} E G^{D-1}}{3(3-2 D)} \times\left(\frac{2(2-D)}{D \psi^{\frac{2-D}{2}}} A_{r}\right)^{\frac{3-2 D}{2}}-\left(2 a_{c}\right)^{\frac{3-2 D}{2}}\right)+\frac{5.6 Y \cdot 76.4^{\frac{2-D}{2(1-D)}}\left(2 a_{c}\right)^{\frac{2-D}{2}}}{2-D}+ \\
& \left.+\frac{2.8\left(1-76.4^{\frac{2.38-1.88 D}{1-D}}\right) k Y\left(2 a_{c}\right)^{\frac{2-D}{2}}}{3(2.38-1.88 D)}\right)
\end{aligned}
$$

Give the total normal contact load in a non-dimensional form as:

$$
\begin{aligned}
P^{*} & =\frac{D}{2} \psi^{\frac{2-D}{2}}\left(\frac{2(2-D)}{D \psi^{\frac{2-D}{2}}} A_{r}^{*}\right)^{\frac{D}{2}} \cdot\left(\frac{2 \sqrt{\pi} G^{* D-1}}{3(3-2 D)} \times\left(\left(\frac{2(2-D)}{D \psi^{\frac{2-D}{2}}} A_{r}^{*}\right)^{\frac{3-2 D}{2}}-\left(2 a_{c}^{*}\right)^{\frac{3-2 D}{2}}\right)+\right. \\
+ & \left.\frac{5.6 \phi \cdot 76.4^{\frac{2-D}{2(1-D)}}\left(2 a_{c}^{*}\right)^{\frac{2-D}{2}}}{2-D}+\frac{2.8\left(1-76.4^{\frac{2.38-1.88 D}{1-D}}\right) k \phi\left(2 a_{c}^{*}\right)^{\frac{2-D}{2}}}{3(2.38-1.88 D)}\right),
\end{aligned}
$$

where $P^{*}=\frac{P}{E A_{a}}, G^{*}=\frac{G}{\sqrt{A_{a}}}, A_{r}^{*}=\frac{A_{r}}{A_{a}}, a_{c}^{*}=\frac{a_{c}}{A_{a}}, \phi=\frac{Y}{E}$.

When $D=1.5$, the elastic, elastic-plastic, plastic normal contact load can be given as:

$$
\begin{aligned}
& P_{e}=\frac{\sqrt{\pi}}{4} E G^{\frac{1}{2}} \psi^{\frac{1}{4}} a_{l}{ }^{\frac{3}{4}} \ln \frac{a_{l}{ }^{\prime}}{a_{c}{ }^{\prime}}, \\
& P_{p}=5.6 Y \cdot \frac{3}{2} \psi^{\frac{1}{4}} a_{l}{ }^{\frac{3}{4}} \cdot 76.4^{-2} a_{c}{ }^{\frac{1}{4}}, \\
& P_{e p}=\frac{3}{4} \psi^{\frac{1}{4}} a_{l}{ }^{\frac{3}{4}} \frac{2.8\left(1-76.4^{-0.12}\right) k Y a_{c}{ }^{\frac{1}{4}}}{0.18} .
\end{aligned}
$$

We can have the expression of the total normal contact load as following:

$$
\begin{aligned}
P & =P_{e}+P_{p}+P_{e p}= \\
& =\psi^{\frac{1}{4}} a_{l}{ }^{\frac{3}{4}}\left(\frac{\sqrt{\pi}}{4} E G^{\frac{1}{2}} \ln \frac{a_{l}{ }^{\prime}}{a_{c}{ }^{\prime}}+5.6 Y \frac{3}{2} 76.4^{-2} a_{c}{ }^{\frac{1}{4}}+\right. \\
& \left.+\frac{3}{4} \frac{2.8\left(1-76.4^{-0.12}\right) k Y a_{c}{ }^{\frac{1}{4}}}{0.18}\right) .
\end{aligned}
$$

Put the equation (56) into the equation (57), so that we can get the final form: 


$$
P=\psi^{\frac{1}{4}}\left(\frac{2(2-D)}{D \psi^{\frac{2-D}{2}}} A_{r}\right)^{\frac{3}{4}}\left(\frac{\sqrt{\pi}}{4} E G^{\frac{1}{2}} \ln \frac{(2-D) A_{r}}{D \psi^{\frac{2-D}{2}} a_{c}}+5.6 Y \frac{3}{2} 76.4^{-2}\left(2 a_{c}\right)^{\frac{1}{4}}+\frac{3}{4} \frac{2.8\left(1-76.4^{-0.12}\right) k Y\left(2 a_{c}\right)^{\frac{1}{4}}}{0.18}\right)
$$

Give the total normal contact load in a non-dimensional form as:

$$
P^{*}=\psi^{\frac{1}{4}}\left(\frac{2(2-D)}{D \psi^{\frac{2-D}{2}}} A_{r}^{*}\right)^{\frac{3}{4}}\left(\frac{\sqrt{\pi}}{4} G^{* \frac{1}{2}} \ln \frac{(2-D) A_{r}^{*}}{D \psi^{\frac{2-D}{2}} a_{c}^{*}}+5.6 \phi \frac{3}{2} 76.4^{-2}\left(2 a_{c}^{*}\right)^{\frac{1}{4}}+\frac{3}{4} \frac{2.8\left(1-76.4^{-0.12}\right) k \phi\left(2 a_{c}^{*}\right)^{\frac{1}{4}}}{0.18}\right)
$$

where $P^{*}=\frac{P}{E A_{a}}, G^{*}=\frac{G}{\sqrt{A_{a}}}, A_{r}^{*}=\frac{A_{r}}{A_{a}}, a_{c}{ }^{*}=\frac{a_{c}}{A_{a}}, \phi=\frac{Y}{E}$.

Because $K_{n}{ }^{*}$ and $P^{*}$ are the function of $A_{r}{ }^{*}$, based on the equation (47),(53),(59), we build the implicit function relationship of $K_{n}{ }^{*}$ and $P^{*}$, which is a complicated non-linear relationship.

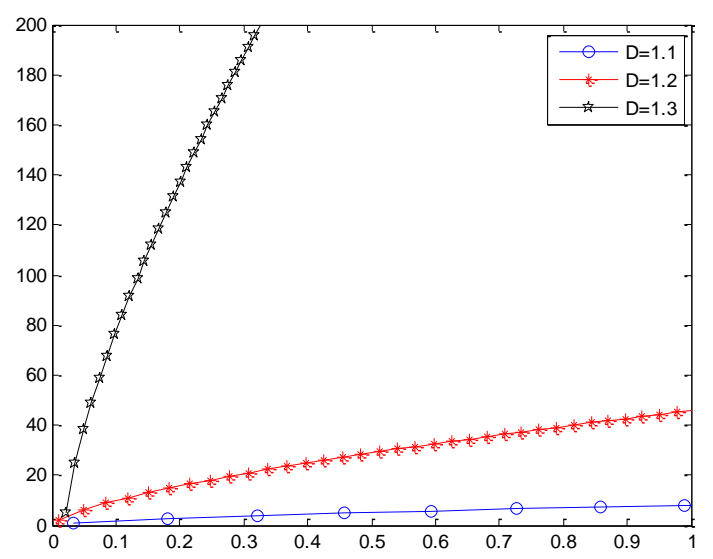

Fig. 3 The change law of total dimensionless normal contact stiffness $K_{n}{ }^{*}$ with total dimensionless contact load $P^{*}$ and the fractal dimension's ( $D$ take1.1,1.2 and 1.3) impact on the total dimensionless normal contact stiffness $K_{n}^{*}\left(G^{*}=1.0 E-10, \phi=1.5\right)$

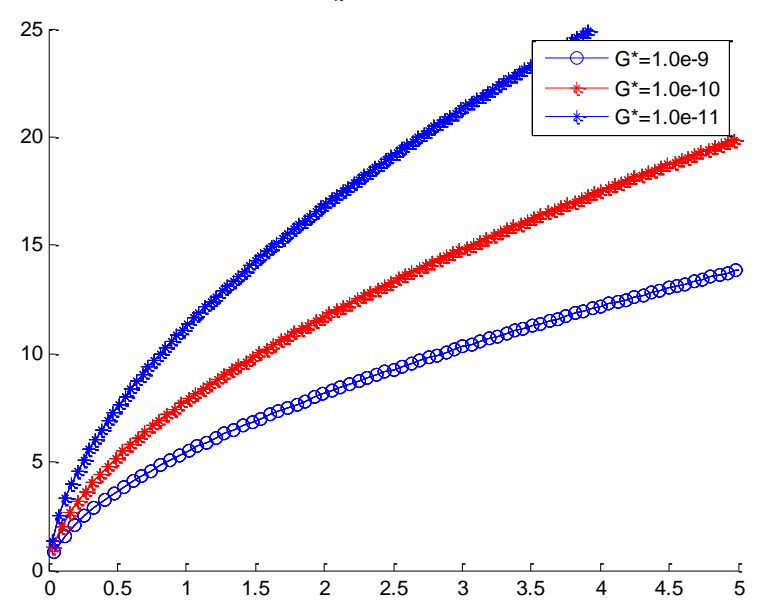

(a) $D=1.1$

\section{The digital simulation of joint surface's normal contact stiffness fractal model}

In this thesis, we give the simulation for the case when $R_{1}=R_{2}$ and the related materials parameters $(\phi, v, Y, E)$ are settled, with fractal dimension $D$ and fractal roughness parameters $G$ are given.

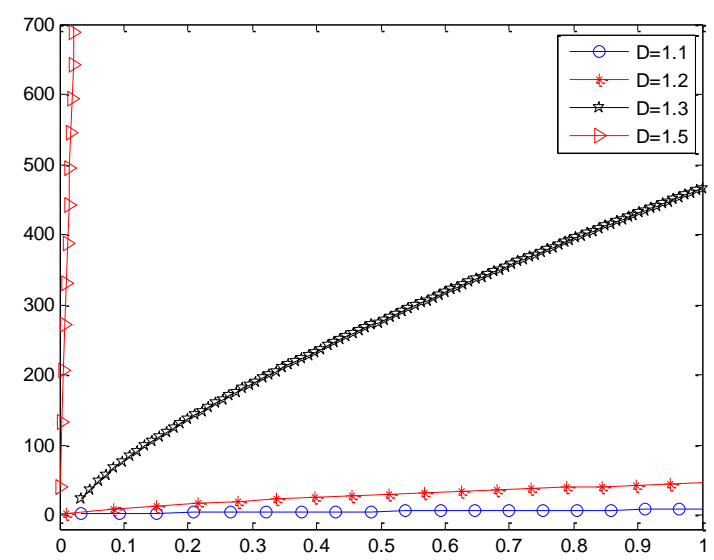

Fig. 4 The fractal dimension's ( $D$ take $1.1,1.2$ and 1.3 ) impact on the total dimensionless normal contact stiffness $\left(G^{*}=1.0 E-10, \phi=1.5\right)$

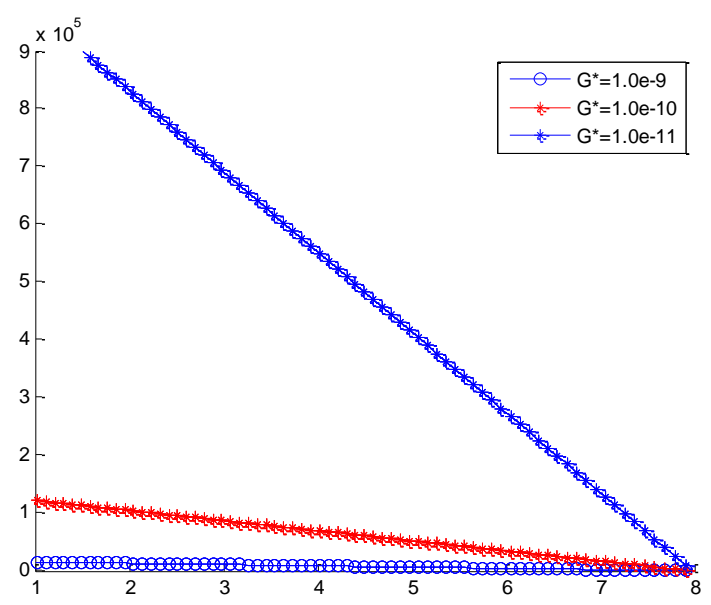

(b) $D=1.9$

Fig. 5 The fractal roughness parameters $G^{*}$ 's impact on the total dimensionless normal contact stiffness $K_{n}{ }^{*}\left(G^{*}=1.0 E-10\right)$ 


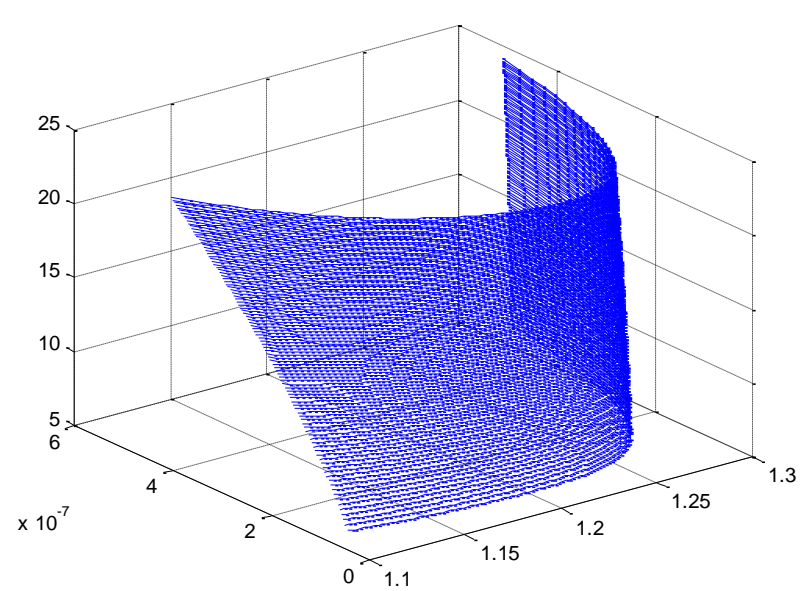

Fig. 6 The three-dimensional plot of total dimensionless normal contact stiffness $K_{n}{ }^{*}$, total dimensionless contact load $P^{*}$ and the fractal dimension's (D take1.1,1.2 and 1.3) impact on the total dimensionless normal contact stiffness $K_{n}{ }^{*}$

At this point is known by the formula (47), (53), (59) that total dimensionless contact load $P^{*}$ and total dimensionless normal contact stiffness $K_{n}{ }^{*}$ are both complex functions of dimensionless real contact area $A r^{*}$. Therefore, according to the literature [19], we take plasticity index $\phi=0.7,1.5,2.5$; Poisson's ratio $v=0.29$,elastic modulus $E_{1}=E_{2}=2.07 \times 10^{11}$, dimensionless roughness parameters $G^{*}=1.0 E-9,1.0 E-11, v_{1}=v_{2}$,fractal dimension $D=1.1 \sim 1.9$. Figs. 3 to 6 are, respectively, given by the change law of total dimensionless contact load $P^{*}$,the simulation results of fractal dimension D's, fractal roughness parameters $G^{*}$ 's plasticity index $\phi$ 's impact on normal contact stiffness considered over elastic-plastic deformation mechanism.

\section{The analysis of simulation results}

1. It can be seen from Fig.3, under the consideration of elastic-plastic transition mechanism, when the fractal dimension $D=1.1 \sim 1.9$, total dimensionless normal contact stiffness $K_{n}{ }^{*}$ increases with the increasing of the total dimensionless contact load $P^{*}$ it is non-linear relationship.

2. According to Fig. 3, total dimensionless normal contact stiffness $K_{n}{ }^{*}$ changes with the total dimensionless contact load $P^{*}$ and the fractal dimension's influence on total dimensionless normal contact stiffness ,we select fractal dimension $D=1.1, D=1.9$ respectively, and simulate the fractal roughness parameters $G^{*}$ and plasticity index $\phi$ 's impact on the total dimensionless normal contact stiffness. The total dimensionless normal contact stiffness $K_{n}{ }^{*}$ increases with the increasing of fractal roughness parameters $G^{*}$, in Fig. 5, a. The total dimensionless normal contact stiffness increases with the plasticity index $\phi$ 's in-

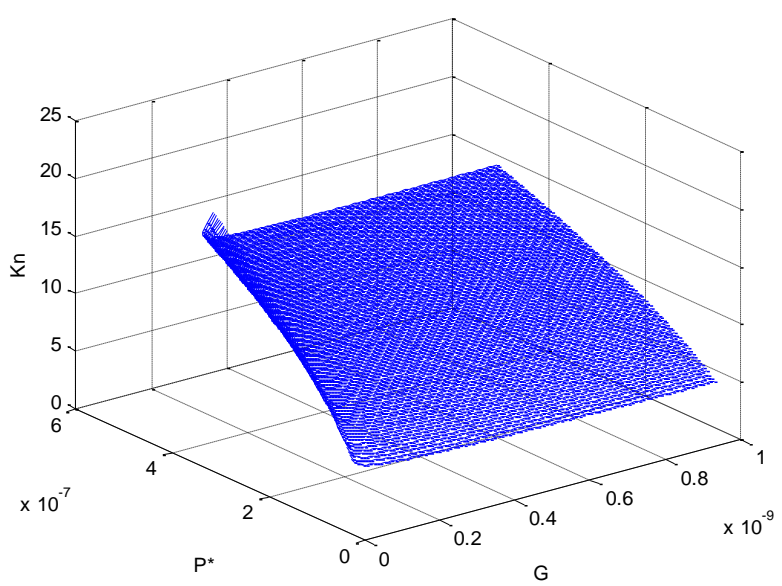

Fig. 7 The three-dimensional plot of total dimensionless normal contact stiffness $K_{n}{ }^{*}$, total dimensionless contact load $P^{*}$ and the fractal roughness parameters $G^{*}$ 's impact on the total dimensionless normal contact stiffness $K_{n}{ }^{*}(\phi=1.5)$

creasing, in Fig. 5, a. But when $D=1.9$, the total dimensionless normal contact stiffness $K_{n}{ }^{*}$ decreases with the increasing of fractal roughness parameters in Fig.5, b; the total dimensionless normal contact stiffness decreases with the plasticity index $\phi$ 's increasing.

3. According to Fig. 6, when the fractal dimension $D=1.1 \sim 1.9$, total dimensionless normal contact stiffness $K_{n}{ }^{*}$ increases with the increasing of total dimensionless contact load $P^{*}$ it is non-linear relationship, which gains the same result with Figs. 3 and 4.

4. According to Fig. 7, the total dimensionless normal contact stiffness $K_{n}{ }^{*}$ increases with the increasing of fractal roughness parameters $G^{*}$, which gains the same results with Fig. 5, a and b.

\section{Acknowledgements}

This work was financially supported by National university student innovation project (201410504066, 20150504077). Thanks to Prof. Jiajun Yang, he is the corresponding author.

\section{References}

1. Greenwood, J A.; Williamson, J B P. 1966. Contact of nominally flat surfaces, Mathematical and Physical Sciences, 295(87): 300-319. http://dx.doi.org/10.1098/rspa.1966.0242.

2. Majumdar, A.; Tien, C.L. 1990. Fractal Characterization and Simulation of Rough Surfaces, Wear 136(2):313-327. http://dx.doi.org/10.1016/0043-1648(90)90154-3.

3. Majumdar, A.; Bhushan, B. 1990. Role of fractal geometry in roughness characterization and contact mechanics of surfaces, ASME Journal of Tribology, 112 (2): 205-216. http://dx.doi.org/10.1115/1.2920243.

4. Wang, S.; Komvopoulos, K. 1994. Fractal theory of the interfacial temperature distribution in the slow sliding 
regime:Part I-elastic contact and heat transfer analysis, ASME Journal of Tribology, 116(4): 812-818. http://dx.doi.org/10.1115/1.2927338.

5. Xueliang, Zhang.; Yumei, Huang.; Ying, Han. 2000. Fractal model of the normal contact stiffness of machine joint surfaces based on the fractal contact theory, China mechanical engineering, 11(7):727- 729.

http://dx.doi.org/10.3321/j.issn:1004-132X.2000.07. 003.

6. Shuhua, Wen.; Xueliang, Zhang.; Meixian, Wu.; Xiaoguang, Wen.; Pengyun, Wang. 2009. Fractal model and simulation of normal contact stiffness of joint interfaces and simulation, Journal of agricultural machinery, 40 (11):197-202.

7. Ganti, S.; Bushan, B.1995. Generalized fractal analysis and its applications to engineering surfaces, Wear, 180(1-2):17-34. http://dx.doi.org/10.1016/0043-1648(94)06545-4.

8. Wang, S.; Konvopoulos, K. 1995. Fractal theory of temperature distribution at elastic contacts of fast sliding surfaces, Journal of Tribology, 117(2): 203-215. http://dx.doi.org/10.1115/1.2831227.

9. He, L.; Zhu, J. 1997. The fractal character of processed metal surfaces, ASME J. Tribol, 208(1-2): 17-24. http://dx.doi.org/10.1016/S0043-1648(96)07330-9.

10. Shuyun, Jiang.; Yunjian, Zheng.; Hua, Zhu. 2010. A contact stiffness model of machined plane joint based on fractal theory, Journal of Tribology, 132(1): 1-7. http://dx.doi.org/ 10.1115/1.4000305.

11. Ghafoor, A.; Dai, J, S.; Duffy, J. 2004. Stiffness modeling of the soft-finger contact in robotic grasping, ASME Journal of Tribology, 646(11): 135-140. http://dx.doi.org/10.1115/1.1758255.

12. Wu, J. J. 2001. The properties of asperities of real surface, ASME Journal of Tribology, 394(11): 1946-1950. http://dx.doi.org/10.1115/1.1353179

13. Robert, L. J.; Jeffrey, L. S. 2006. A multi-scale model for contact between rough surfaces, Wear, 261(11-12): 1337-1347.

http://dx.doi.org/10.1016/j.wear.2006.03.015.

14. Persson, B. N. J. 2006. Contact mechanics for randomly rough surface, Surface Science Reports, 61(3): 201-227.

http://dx.doi.org/10.1016/j.surfrep.2006.04.001.

15. Dickrell, D. J.; Sawyer, W. G. 2011. Lateral contact stiffness and the elastic foundation, Tribology Letters, 41(1): 17-21.

http://dx.doi.org/10.1007/s11249-010-9666-5.

16. Filippi, S.; Akay, A.; Gola, M. M. 2004. Measurement of tangential contact hysteresis during microslip, ASME Journal of Tribology, 482(5): 78-85. http://dx.doi.org/10.1115/1.1692030.

17. Kartal, M. E.; Mulvihill, D. M.; Nowell, D.; Hills, D. A. 2011. Measurements of pressure and area dependent tangential contact stiffness between rough surfaces using digital image correlation, Tribology International, 44(21): 1188-1198. http://dx.doi.org/10.1016/j.triboint.2011.05.025.

18.Shuhua, Wen.; Xueliang, Zhang.; Meixian, Wu.; Xiaoguang, Wen.; Pengyun, Wang. 2009. Combining with the surface normal contact stiffness of fractal model is established and simulation, Journal of agricultural machinery, 40(11): 197-202.
19. Nashan, Wang; Xueliang, Zhang; Guosheng, Lan; Shuhua, Wen; Yonghui, Chen; Liqin, Liu; Zuozheng, Niu. 2014. The critical parameters of continuous contact elastic-plastic fractal rough surface normal contact stiffness model, Journal of vibration and shock, 33(9): 72-77.

http://dx.doi.org/10.13465/j.cnki.jvs.2014.09.013.

20. Xueliang, Zhang; Meiyu, Huang; Weiping, Fu; Wenpeng, Zhang. 2000. The fractal model of rough surface normal contact stiffness, Journal of applied mechanics, 17(2): 31-36.

http://dx.doi.org/10.3969/j.issn.1000-4939.2000.02.006.

21. Xueliang, Zhang; Meiyu, Huang; Ying, Han. 2000. The method of machine joint surface is based on the theory of the fractal contact to contact stiffness model, Chinese mechanical engineering, 11(7): 727-730. http://dx.doi.org/10.3321/j.issn:1004132X.2000.07.003.

22. Xueliang, Zhang. 2002. The dynamic characteristics of machine joint surface and its application. Beijing: China science and technology publishing house: 1-2.

23. Shuhua, Wen; Xueliang, Zhang; Meixian, Wu. 2009. Fractal model of normal contact stiffness of joint interfaces and its simulation, Journal of agricultural machinery, 40(11): 197-202.

24. Huiguang, Li; Heng, Liu; Lie, Yu. 2011. Contact stiffness of rough mechanical joint surface, Journal of xi 'an jiaotong university, 45 (6): 69-74. http://dx.doi.org/10.7652/xjtuxb201106013.

25. Li, Huiguang; Liu, Heng.; Yu, Lie. 2012. Dynamic characteristics of rod fastening rotor for gas turbine considering contact stiffness, Journal of vibration and shock, 31(7): 4-8. http://dx.doi.org/10.3969/j.issn.1000-3835.2012.07.002.

26. Zhushi, Rao; Songbo, Xia; Guangming, Wang. 1994. A study of contact stiffness of flat rough surfaces. Journal of Mechanical Strength, 16(2): 72-75.

27. Yanting, Yi ; Xue, Zhai; Zhi, Wang. 2012. Influences of normal contact stiffness on an assembly's vibration models, Journal of vibration and shock, 31(6): 171-174. http://dx.doi.org/10.3969/j.issn.1000-3835.2012.06.035.

28. Yi, Liu.; Heng, Liu.; Jun,Yi. 2013. Equivalent method for normal contact stiffness considering plastic contact layer, Journal of vibration and shock, 32(7): 43-47. http://dx.doi.org/10.3969/j.issn.1000-3835.2013.07.009.

29. Xueliang, Zhang.; Yumei, Huang.; Ying, Han. 2000. Fractal model of the normal contact stiffness of machine joint surfaces based on the fractal contact theory, China mechanical engineering, 11(7): 727-729.

http://dx.doi.org/10.3321/j.issn:1004132X.2000.07.003.

30. Guosheng, Lan.; Xueliang, Zhang.; Hongqin, Ding. 2011. Modified contact model of joint interfaces based on fractal theory, Transactions of the Chinese Society for Agricultural Machinery, 42(10): 217-223. http://dx.doi.org/10.3969/j.issn.1000-1298.2011.10.042.

31. Shuyun, Jiang.; Yunjian, Zheng. 2010. A contact stiffness model of machined joint surfaces, Journal of Tribology Transactions of ASME, 132(1): 1-7. http://dx.doi.org/10.14257/ijca.2014.7.6.03.

32. Kogut, L.; Etsion, I. 2002. Elastic-plastic contact analysis of a sphere and a rigid flat, American Society of Mechanical Engineers, 69(5): 657-662. http://dx.doi.org/10.1115/1.1490373.

33. Xueliang, Zhang.; Yumei, Huang.; Shuhua, Wen. 2000. Fractal model of contact stiffness of joint surface, 
Transactions of the Chinese Society for Agricultural Machinery, 31(4): 89-91.

34. Xueliang, Zhang.; Shuhua, Wen. 2002. A fractal model of tangential contact stiffness of joint surfaces based on the contact fractal theory, Transactions of the Chinese Society for Agricultural Machinery, 33(2): 9193.

35. Xueliang, Zhang.; Shuhua, Wen. The dynamic characteristics of machine joint surface and its application, Science and technology of China press, Beijing.

36. Guangqin, Wang. 2008. Elastic mechanics, China railway publishing house: 183-186.

37. Shuhua, Wen.; Xueliang, Zhang.; Meixian, Wu. 2009. Fractal model of normal contact stiffness of joint interfaces and its simulation, Journal of agricultural machinery, 40(11): 197-202.

38. Liou, J L. 2006. The theoretical study for microcontact model with variable topography parameters, Tiwan: National Cheng Kung University.

39. Jinmin, You.; Tianning, Chen. 2009. Fractal model for normal dynamic parameters of joint surfaces, Journal of Xi'an Jiao Tong University, 43(9): 91-94. http://dx.doi.org/10.7652/xjtuxb200909020.
Jingfang Shen, Sha Xu, Wenwei Liu, Jiajun Yang

FRACTAL MODEL OF NORMAL CONTACT STIFFNESS BETWEEN TWO SPHERES OF JOINT INTERFACES WITH SIMULATION

S u m m a r y

The contact stiffness of joint surface plays a significant role in the overall static and dynamic characteristics of mechanical systems. When considering joint interfaces with two rough surfaces, the traditional model based on Hertz contact theory between a sphere and a plane is difficult to use, especially for increasingly complex engineering surfaces. In order to overcome the weakness, here we propose a new contact stiffness model in view of the influence of domain extension factor between two rough surfaces. We study the deformation mechanism and the critical contact parameters. Subsequently, we analyze evolution of the elastic-plastic contact involving three distinct stages ranged from complete elastic through elastic-plastic to fully plastic deformation. Our fractal model is more universal than the traditional model based on some strict assumptions which simplify the contact of two rough surfaces to a rough surface and a rigid plane. In fact, the traditional model could be regarded as a special case in our new model. Simulations show that non-dimensional normal contact stiffness increases with dimensionless contact total load under the mechanism of elastic-plastic transition when the fractal dimension is between 1.1 to 1.5 . The results indicate that contact stiffness of our fractal model is appropriate and the theoretical contact stiffness is consistent with the experiment data.

Keywords: contact stiffness, fractal model.

Received June 29, 2016

Accepted October 13, 2017 